\title{
Impact of Power Consumption in Sensors Life Time for Wireless Body Area Networks
}

\author{
Lana Ibrahim ${ }^{1}$, Hana Osman ${ }^{2}$, Abdala Osman ${ }^{3}$ and Elmustafa Sayed Ali ${ }^{4}$ \\ Electrical and Electronics Engineering Department, Red Sea University, Sudan \\ elmustafasayed@rsu.edu.sd
}

\begin{abstract}
Wireless body area network (WBAN) is a communication standard optimized for low power devices and operation on, in or around the human body but not limited to humans used to serve a variety of applications including medical, consumer electronics, entertainment and other[1]. The challenge of WBAN is that the energy of the sensors is limited by the battery life that's why the transmitting of data is not continuous for long time. This leads to poor efficiency of the WBAN because the continuous power decreasing is due to network operations. An algorithms such as MAC zigbee802.15.4 and adaptive reliable MAC are important to reduce the replacement of batteries many times and to save power consumption. In this paper we concentrated our study on some type of sensors, they are telos $B$, mica2, micaz, and imote 2 depends on power consumption calculations using mat lab program and based on adaptive reliable MAC. We analyzed the sensors with several nodes and packet sizes, and calculate the lifetimes for all the sensors in WBAN. The results we obtained show a good performance of using Adaptive reliable MAC in Wireless Body Area Networks.
\end{abstract}

Keywords: WBAN, sensors, telosB, mica2, micaz, imote2, MAC 802.15.4 ZigBee, adaptive reliable MAC.

\section{Introduction}

The wireless communication technology and its development have progressed a lot in various fields of study, but none so more than in the field of wireless networks. Among the various research areas and applications of this technologies one of the most applications is Wireless Body Area Network (WBAN), which can be defined as a miniature network consisting of sensors operating mainly on or around the human body as shown in figure 1 below [1]. Technological advancements in devices with features like low cost, low size, ultra-low power consumption and emission, with more reliable communication expand the span of usage scenarios and applications especially in healthcare and monitoring [2]. 


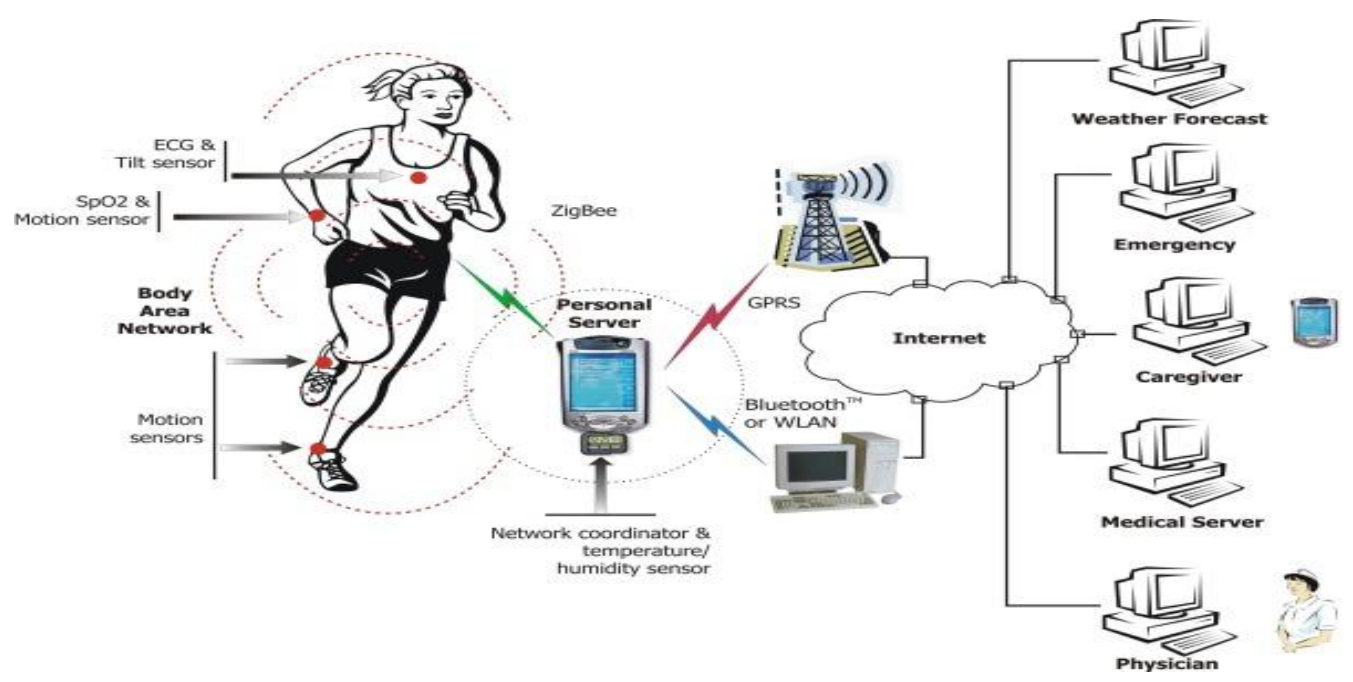

Figure 1. Wireless Body Area Network Model

Power consumption is one of the most important issues in wireless body area networks. The longevity of the WBAN network depends heavily on the battery life cycle of the sensors and actuator nodes. Also to obtain miniature size of sensors, battery sizes should be kept small since their size affects the overall size of the nodes. Energy saving is one of the most efficient ways of ensuring that the WBAN network sensors runs years without any problems. Energy efficiency is an important issue because the power of sensor nodes in WBANs is limited while long duration of operation is expected [3], therefore it must chooses the suitable type of sensor which are needed to WBAN application and must be compatible with the number of nodes and packet size base on sensor power consumption and live time.

\section{Medium Access Control (MAC)}

Medium access control is a solution of the problems of accessing the medium (channel), the problem that facing the accessing such medium is who gets accessing at any given time. This section review a two types of medium access control protocols are used in WBAN they are, ZigBee MAC and Adaptive Reliable MAC.

\subsection{IEEE 802.15.4 ZigBee MAC}

IEEE 802.15.4 is a low-power standard designed for low data rate applications. The IEEE 802.15.4 standard specifies both the physical (PHY) and medium access control (MAC) layers for low power, low data rate and low cost wireless network devices .The PHY layer uses direct sequence spread spectrum (DSSS) and defines different transmission rates [4]. The MAC layer defines two different operation modes: a nonbeacon-enabled mode, which uses an unspotted CSMA/CA (Carrier Sense Multiple Access / Collision Avoidance) algorithm, and a beacon enabled mode, which defines a super frame structure and uses a slotted CSMA-CA algorithm. The MAC layer provides also an optional guaranteed time slot (GTS) scheme, which allows the allocation of dedicated bandwidth for devices; however, this scheme is limited to a maximum of seven GTS allocations. IEEE 802.15.4 has remained the main focus of many researchers during the past few years. Some of the main reasons of selecting IEEE 802.15.4 for WBAN are low-power communication and support of low data rate WBAN applications [5]. 


\subsection{Adaptive Reliable MAC}

Extensive energy is consumed by transceiver communication operation. Existing research on MAC layer focuses to maximize battery-powered sensor node's life. Bottleneck of MAC layer protocol design for WBAN is to achieve high reliability and energy minimization. Majority of MAC protocols designed for WBANs are based upon TDMA approach. However, a new protocol needs to be defined to achieve high energy efficiency, fairness and avoid extra energy consumption due to synchronization. Proposed AR-MAC protocol by A.Rahim et al [6] is based upon TDMA approach to minimize energy consumption. AR-MAC assigns Guaranteed Times Slot (GTS) to each sensor node for communication based upon the requirements of sensor node. To reduce overhearing and idle listening the proposed system uses periodic sleep and wakeup according to node requirements.

Once sensor node selects a proper RF Channel after receiving Channel Packet from central node CN, sensor node sends out a Time Slot Request (TSR) packet to CN. TSR packet includes sensor node's data rate and required time slot information. The proposal time slots of fixed length with fixed guard band time, GBT. In or on-body biomedical sensors have different data rates and sampling intervals with different clock drifts. Adaptive Reliable MAC uses an adaptive scheme for Time Slot (TS) and GBT time. Based on traffic pattern of nodes, $\mathrm{CN}$ assigns time slot and sends Time Slot Request Reply (TSRR). These time slots are of variable length depend upon the requirements of sensor nodes instead of fixed length time slots in ZigBee MAC [6].

\section{Sensors Parameters and Life Time}

There are many types of sensors which they are used in WBAN, the most common sensors are, Micaz, Mica2, TelosB and Imote2 which they produced from Crossbow company and running under Tiny operating system. The parameters of these sensors are shown in the following table.

Table 1. Sensors Parameters Model

\begin{tabular}{|c|c|c|c|c|c|}
\hline Sensors & $\begin{array}{c}\text { Supply } \\
\text { voltage } \\
\text { (volt) }\end{array}$ & $\begin{array}{l}\text { Tx mode } \\
\text { current }(\mathbf{m A})\end{array}$ & $\begin{array}{l}\text { Rx mode } \\
\text { current }(m A)\end{array}$ & $\begin{array}{l}\text { Sleep mode } \\
\text { current }(\mu \mathrm{A})\end{array}$ & $\begin{array}{l}\text { Battery } \\
\text { Capacity } \\
\text { (m Ah) }\end{array}$ \\
\hline$B^{\text {telos }}$ & 3 & 17 & 23 & 5.1 & 2600 \\
\hline $\mathrm{e} 2^{\text {Imot }}$ & 5.5 & 17 & 20 & 390 & 1400 \\
\hline $2^{\text {Mica }}$ & 3.3 & 25 & 8 & 15 & 2000 \\
\hline $\mathrm{z}^{\text {Mica }}$ & 3.3 & 17 & 20 & 15 & 2000 \\
\hline
\end{tabular}

The lifetime of the sensor node (L) can be calculated by the equation (1) below [7], it depends on the capacity of the battery $\left(C_{\text {batt }}\right)$ and the total energy consumed by the battery $(\mathrm{E})$ in addition to the value of supply voltage (v).

$$
\mathrm{L}=\frac{C_{b a t t * V}}{E}
$$


Where:

$\mathrm{L} \equiv$ the lifetime of the node $(\mathrm{sec}), C_{\text {batt }} \equiv$ Capacity of the battery $(\mathrm{m} \mathrm{Ah}), \mathrm{V} \equiv$ value of supply (volt), and $\mathrm{E} \equiv$ energy consumption (mille joule).

\section{Simulations Models}

In this study we simulates the WBAN by using Matlab to evaluate the performance of sensors under the operation of IEEE802.15.4 ZigBee and AR-MAC based power consumption due to packets drop rate . The simulation environment is based on many WBAN parameters included values such as listening mode current ,supply voltage, transmitter current, receive current, sleep mode current. Our proposed study will be divided into three scenarios. Scenario one represents a performance of sensor using different number of nodes such as $5,10,15$, and 20, based on packet size increasing from 50 to 200 bytes to evaluate the operation of using AR-MAC. Scenario two illustrate the performance of sensors in the AR-MAC protocol using different packets sizes such as 50, 100,150 , and 200 byte based on number of nodes increased from 5 to 20 nodes. Scenario three represents the calculation of the sensors life time in lower and higher power consumption using AR-MAC in WBAN .The simulated networks parameters are shown in the following table.

Table 2. Simulated Network Parameters

\begin{tabular}{|c|c|}
\hline Parameter & Type \\
\hline Network & WBAN \\
\hline MAC protocol & 802.15 .4 and AR-MAC \\
\hline Number of sensors & $5,10,15,20$ \\
\hline Packets sizes & $50,100,150,200$ bytes \\
\hline Sensors & Micaz,telosB,Imote2,Mica2 \\
\hline
\end{tabular}

\section{Simulation and Results Discussion}

The network was simulated depending on a number of values that have been adapted based on WBAN and adaptive reliable MAC. The simulation results obtained will be discussed in three scenarios as follows.

\subsection{Scenario 1}

In this section the study of sensors with 5 nodes, 10 nodes, 15 node and 20 nodes is carried out with different packet sizes 50,100,150,200 bytes to evaluate the performance of AR-MAC. The output results are shown in the fallowing figures. 


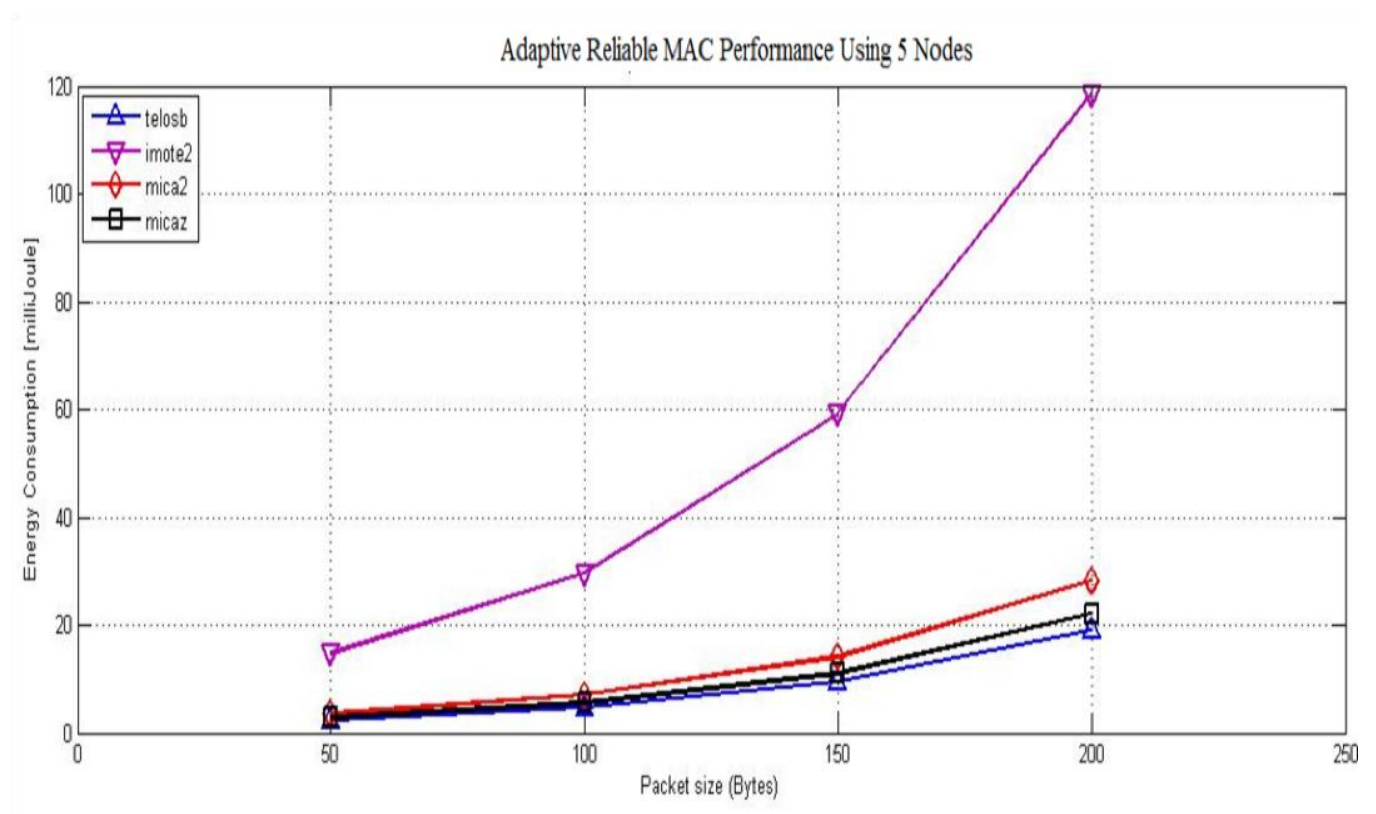

Figure 1. Sensors Energy Consumption with 5 Nodes

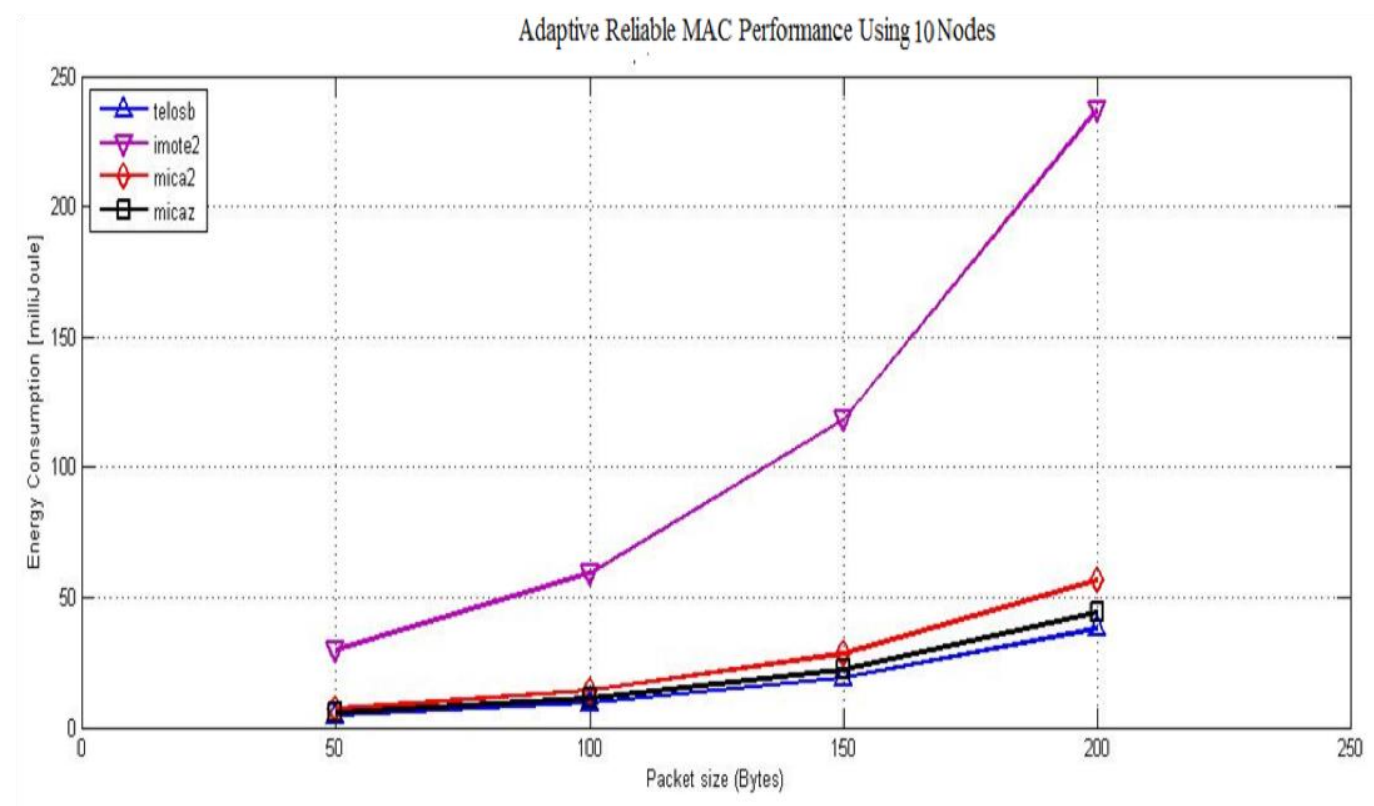

Figure 2. Sensors Energy Consumption with 10 Nodes 


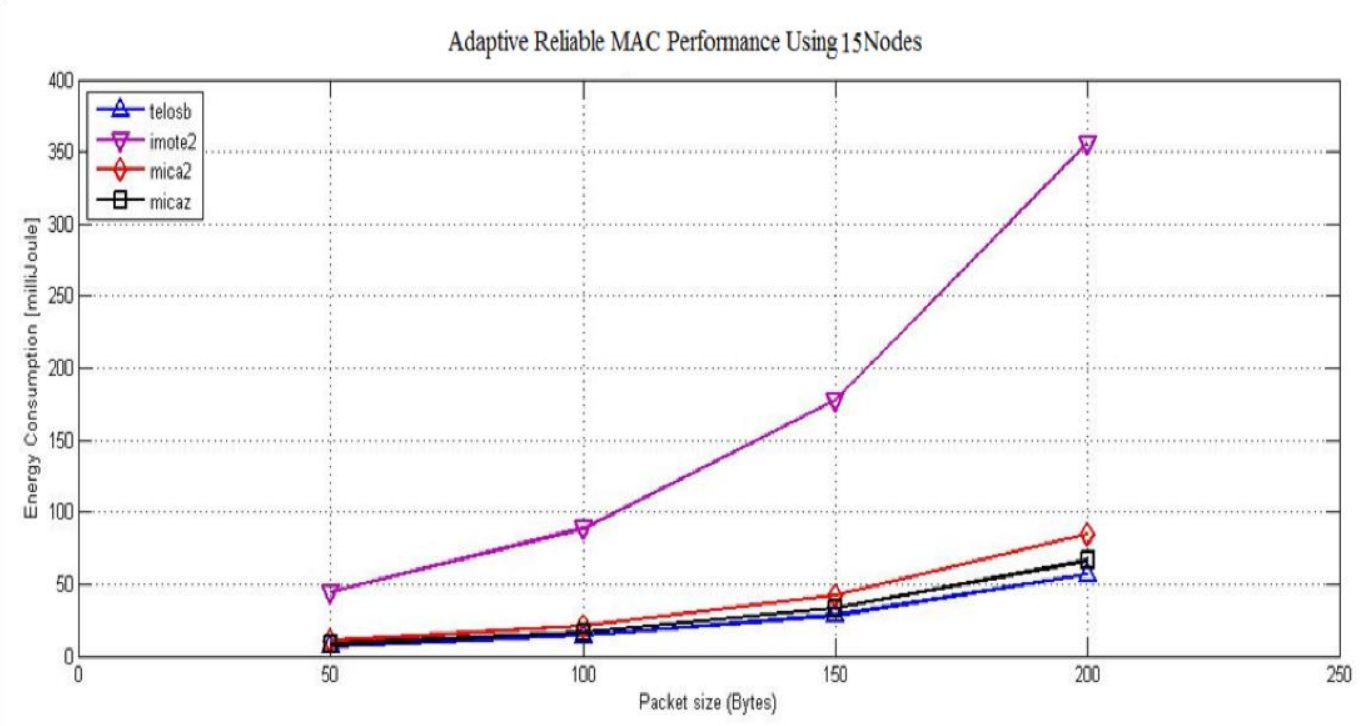

Figure 3. Sensors Energy Consumption with 15 Nodes

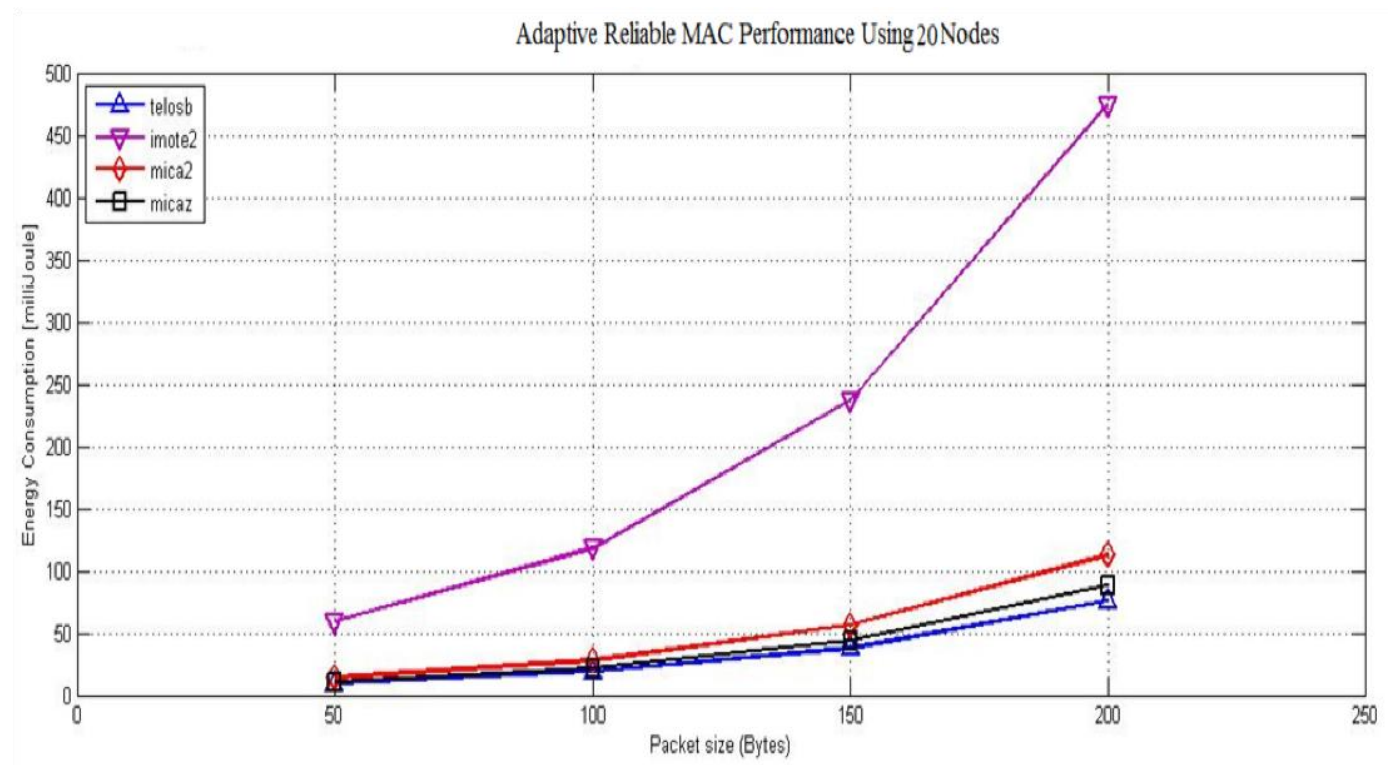

Figure 4. Sensors Energy Consumption with 20 Nodes

In figure 1, it's clear that with 5nodes, the energy consumption increases while packet size increased. Imote 2 consumed approx. 18 mille joules and all other sensors consumed only 5 mille joule in 50 bytes. By increasing in packet size to 200 bytes, Imote 2 will consume approx. 120 mille joules and other sensors have closed power consumption between 20 to 40 mille joules. Figure 2, shows the performance of AR-MAC at 10 nodes. From figure 2 we notice that energy consumption of Imote 2 increased by increasing in numbers of nodes. While packet size increases to 50 bytes, the energy consumption remains approx. to 45 mille joules, and with 200 bytes power consumption becomes 240 mille joule. Other sensors in 50 bytes consumed approximately between 5 to 15 mille joules, and with 200 bytes Mica2, Micaz and TelosB sensors consumed 51, 48, and 47 mille joules respectively. In figure 3 , the performance of 15 nodes show that Imote 2 consume 50 mille joule in 50 bytes and 350 in 200 bytes. Other sensors consumed 
approximately between 5 to 15 mille joule in 50 bytes and between 50 to 100 in 200 bytes, the consumption values are, Mica2 consumed 95, micaz 70, telosb55 mille joules. The performance at 20 nodes in figure 4, show that Imote 2 consumed approx. 60 mille joule in 50 bytes, and 460 mille joules in 200 packets bytes. With 200 bytes Mica 2 consumed 110, TelosB 80 and Micaz 48 milli joules. From scenario 2, it's clear that when the packet size decreases the sensors consume low energy, and with increasing of packet sizes the energy consumption will be increased. Note that in Imote 2 when the number of packet becomes small the Imote 2 sensor consumes high power than another sensor and becomes higher with more packet sizes. With 50 bytes the power consumption values of sensors Mica2, Micaz, TelosB are approximately close to each other, but with 200 bytes, Mica2 consumes higher energy consumption than Micaz, TelosB, and Imote2.

\subsection{Scenario 2}

In this scenario the performance of sensors based on AR-MAC is presented with packet sizes 50,100, 150, 200 bytes, aby using different number of nodes between 5 to 20 nodes. The output obtained as in fallowing figures.

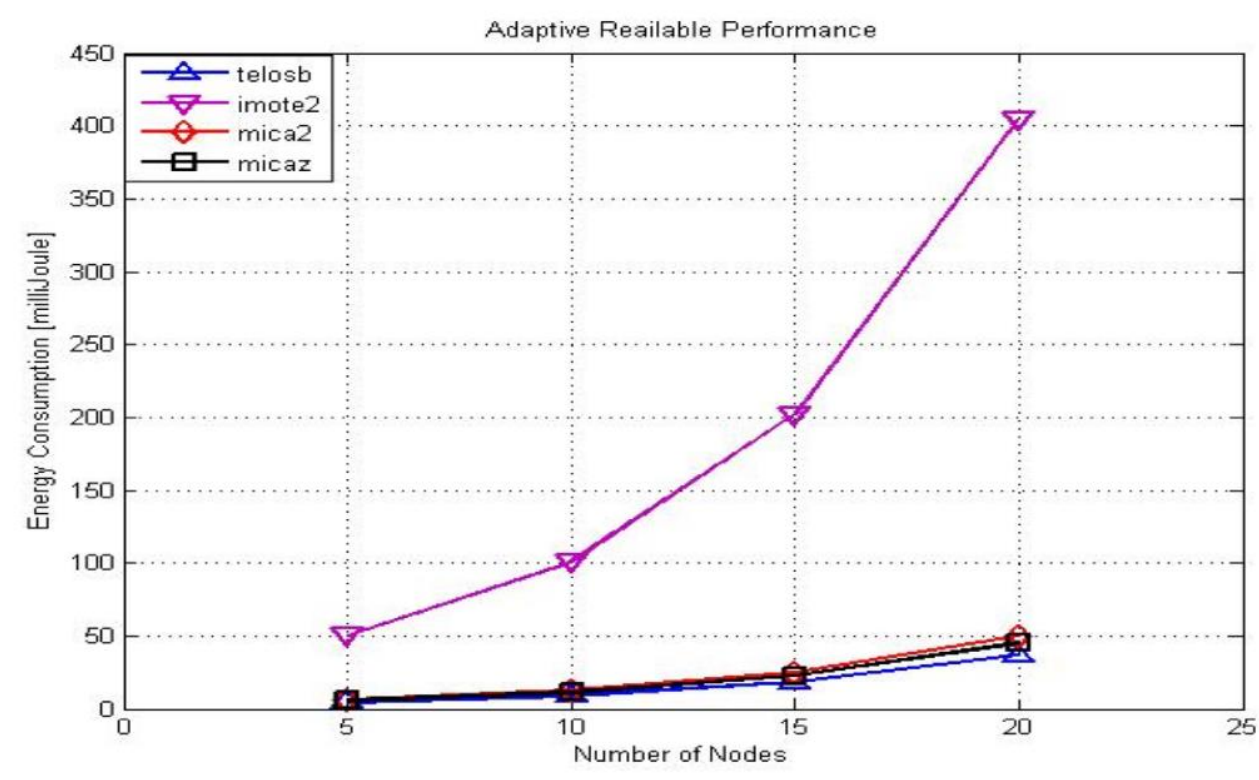

Figure 5. Sensors Energy Consumption with 50 Bytes 


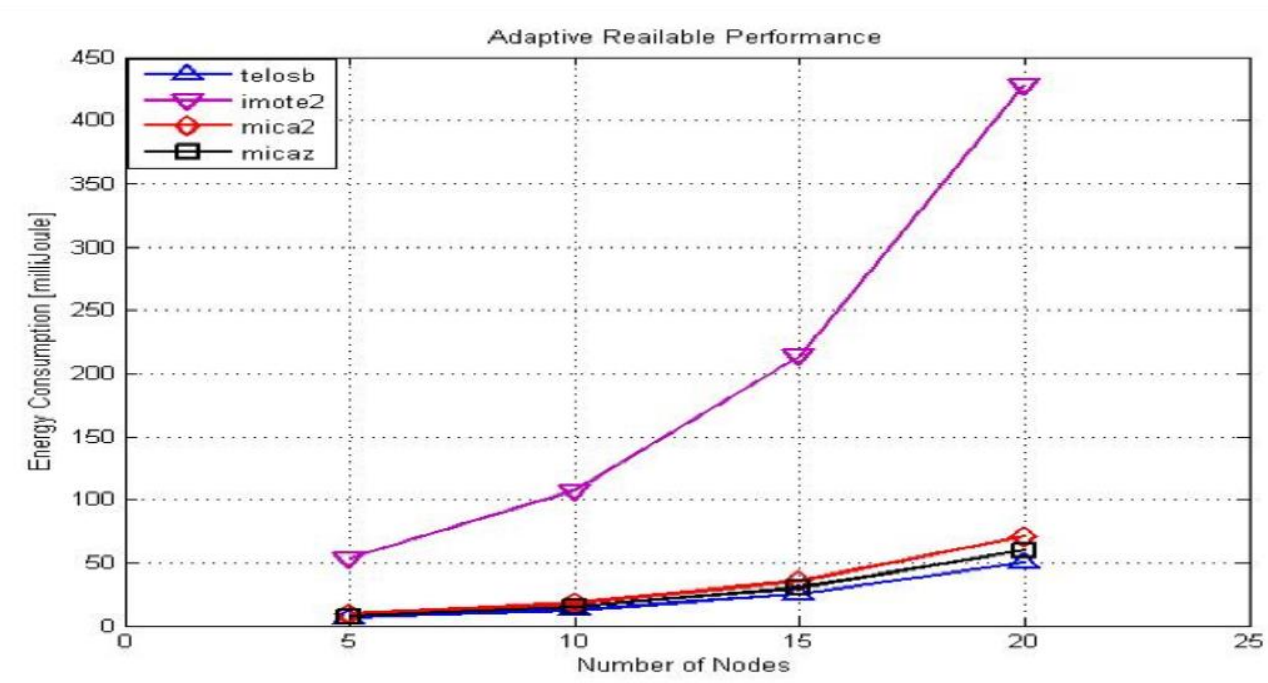

Figure 6. Sensors Energy Consumption with100 Bytes

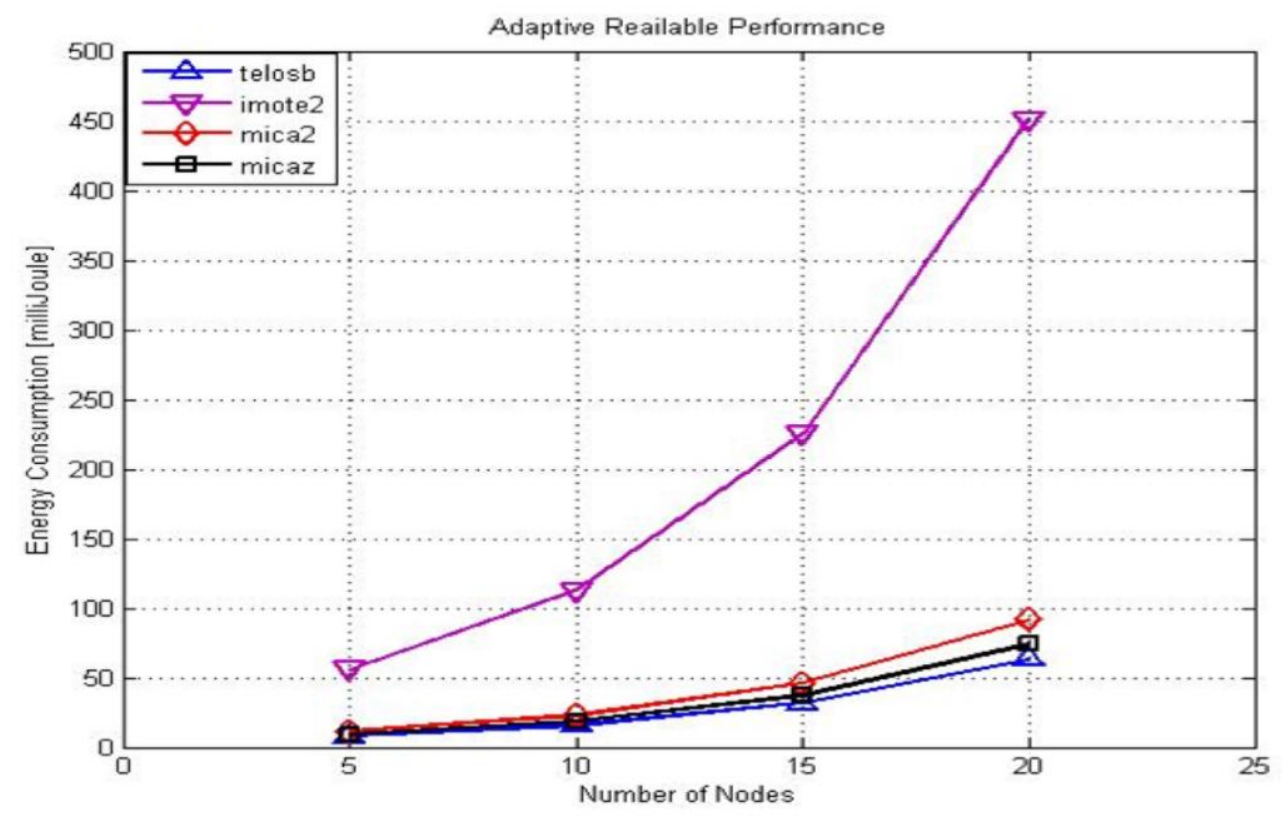

Figure 7. Sensors Energy Consumption with 150 Bytes 


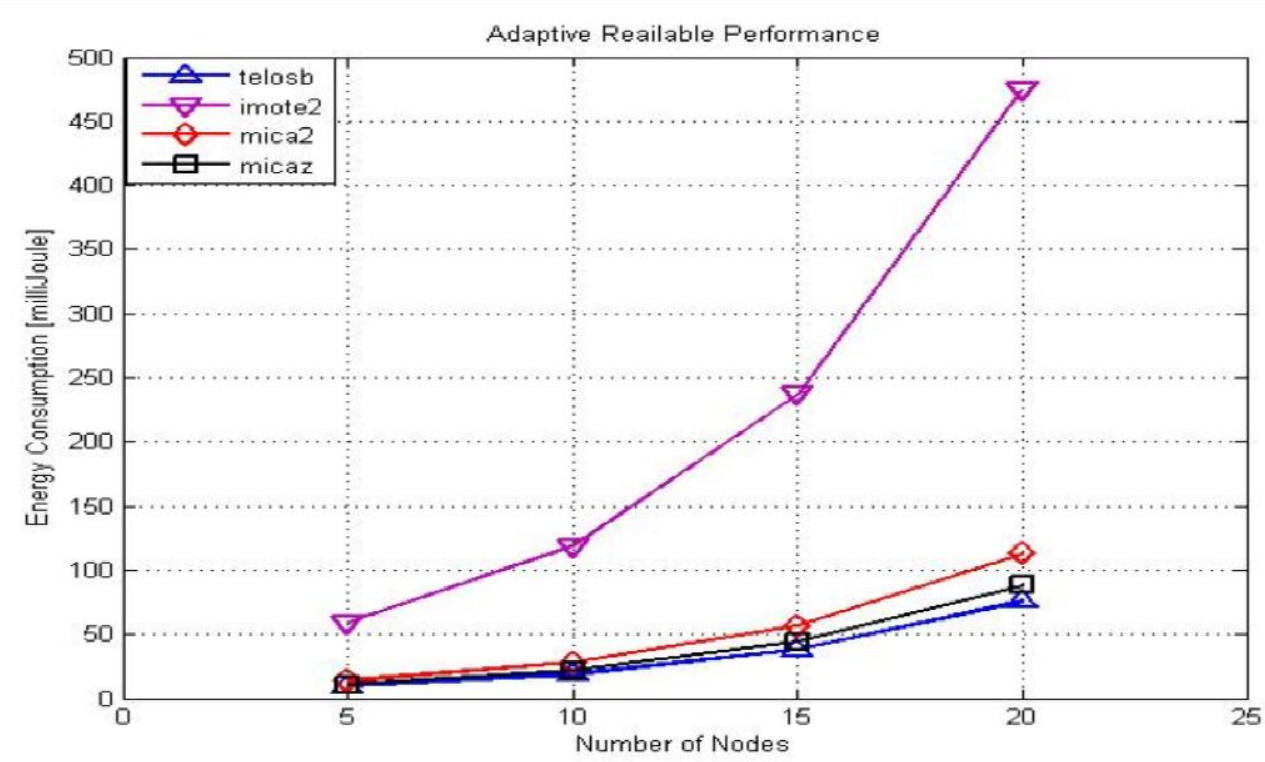

Figure 8. Sensors Energy Consumption with 200 Bytes

Figure 5 shows the AR-MAC sensors performance in packet size 50 bytes. The energy consumption increases due to the increasing in number of nodes. Imote 2 consumed high energy than other sensors. It consumed 50 mille joules in 5 nodes, and 400 mille joules in 20 nodes. Mica2,Micaz,TelosB consumed only 5 mille joule in 5 nodes .with 20 nodes Mica2, Micaz, and TelosB consumed 50, 49, and 47 respectively . In Figure 6 with 100 bytes. Imote 2 consumed 51 mille joules in low number of nodes, and consumed 430 mille joules in when the number of nosed increased. Mica2, Micaz, TelosB consumed 5 mille joule in 50 bytes, and they consume 60, 55, 50 mille joule respectively.

Figure 7 shows the performance when using 150 bytes. Imote 2 consumed 51 mille joule in low number of nodes and 450 mille joules when the number of nodes becomes higher. All other sensors Mica2, Micaz, TelosB consumed 5 mille joule when using 5 nodes, and with 20 nodes they consumed 98, 80, 60 mille joules respectively. In Figure 8, the performance of AR-MAC sensors show that Imote 2 gives the same performance as in figures 5, 6, and 7. With 20 nodes Imote 2 consumed 460 mille joule. Other sensor consumed only 5 mille joules, and with 20 nodes Mica2 consumed 110, Micaz 98, TelosB 70 milli joules. From scenario three, it's clear that when the number of nodes become small the sensor consumes low energy. Imote 2 in small node consumes high energy than another sensors as for increase the number of node it consumed more energy than another sensors.

\subsection{Scenario 3}

In scenario 3, we calculate the lifetime of the sensors per day and then obtain the low and high power consumption of sensors due to lifetime. The results we obtained are shown in figures 9 and 10 below. 


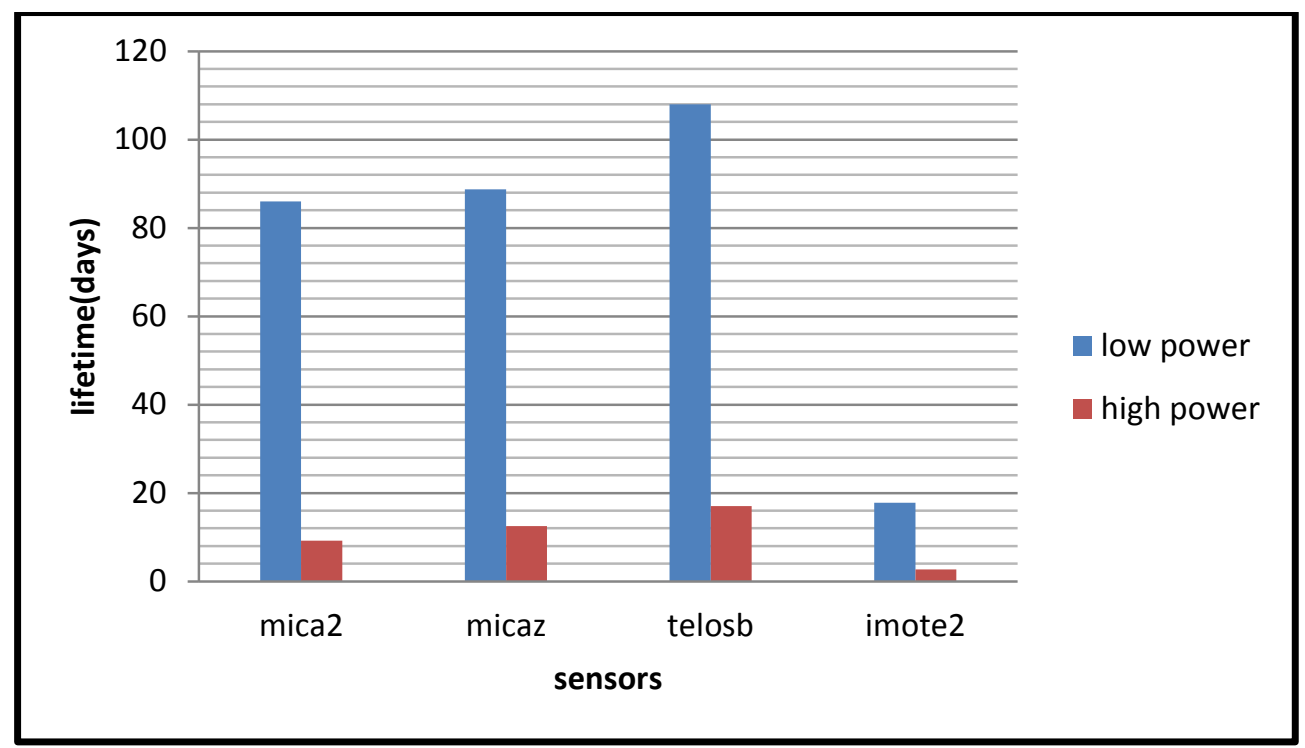

Figure 9. Sensors Lifetime using 5 Nodes

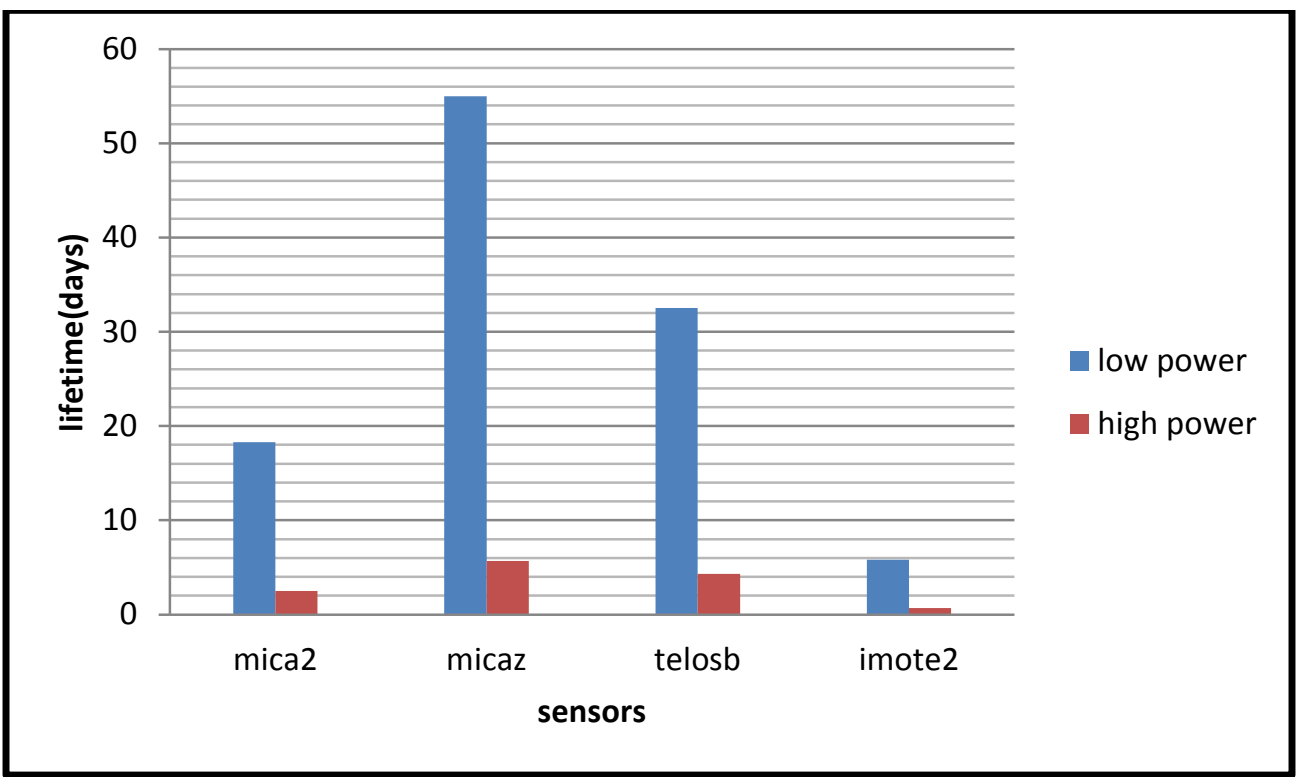

Figure 10. Sensors Lifetime Using 20 Nodes

From figures 9 and 10; it's clear that sensors have lifetime longer in low power consumption and vice versa. The better sensor lifetime is obtained by Micaz, and the other sensors remains an acceptable lifetime respectively as follows; TelosB, Mica2, and Imote2. The lifetime of all sensors depends on the size of packets processed in the sensor model, which means that a high data rate requires more operations and it will consume more power which will lead to low sensors lifetime.

\section{Conclusion}

In this paper, the sensors energy consumption of WBAN has been analyzed and evaluated under the control of adaptive reliable MAC protocol. The analysis and evaluation is based on different numbers of nodes and packet sizes. The evaluated sensors are TelosB, Mica2, Micaz, and Imote2. In our study we analyzed the sensors in WBAN 
consists a different numbers of nodes with changing in packet size, and also we evaluate the WBAN sensors in different packet sizes based on different number of nodes used in the network, in addition to calculations of all sensors lifetimes. The results we are obtained that all sensors work with low power consumption have a long lifetime compared to work with high power consumption which have short lifetime, this fact is because of amount of data processed in the sensors processor. Since adaptive reliable MAC is provided to reduce the process energy consumption in the ZigBee MAC by reduce overhearing and idle listening energy but it still suffering from power consumption due to a large amount of packet sizes processed by the sensors. Although adaptive reliable MAC uses periodic sleep and wakeup according to node requirements which will reduce the idle listening energy but the large amount of data will make sensors almost in a permanent weak up mode which leads to an increase in energy consumption and this of course depends on the different energy consumption levels of sensors. The life time results we obtained from simulation is that Imote 2 had the worst lifetime compared with the others sensors because it had low life time period in low energy consumption compared with the others sensors and very low lifetime period in high power energy consumption. The best lifetime with high data rate and number of nodes is given by Micaz, and TelosB sensors, which they give a better lifetime with lower power consumption.

\section{References}

[1] Erik Karulf," Body Area Networks (BAN)”, http://www.cse.wustl.edu/ jain/cse57408/ftp/ban/index.html. [Accessed in 12 Sep 2015].

[2] Rupinder Kaur," Wireless Body Area Network \& ITS APPLICATION", an International Journal of Engineering Sciences ISSN: 2229-6913 Issue July 2011, Vol. 1.

[3] Sidharth Nabar_, Ayan Banerjeey, Sandeep K.S. Guptayand Radha Poovendran_, Evaluation of Body Sensor Network Platforms: A Design”, University of Washington, Seattle, USA, Oct 18, 2014.

[4] Sai Anand Gopalan, Jong-Tae Park, Energy-Efficient MAC Protocols for Wireless Body Area Networks", Ultra-Modern Telecommunications and Control Systems and Workshops (ICUMT), 2010

[5] Sana Ullah, Bin Shen, etc., "A Study of Medium Access Control Protocols for Wireless Body Area Networks" arXiv: 1004.3890v2 [cs.NI] 31 Jul 2010.

[6] A. Rahim, N. Javaid, M. Aslam, U. Qasim, Z. A. Khan," Adaptive Reliable Medium Access Control Protocol for Wireless Body Area Networks", arXiv:1207.3599v1 [cs.NI] 16 Jul 2012.

[7] Jyoti Saraswat, and Partha Pratim Bhattacharya," EFFECT OF DUTY CYCLE ON ENERGY CONSUMPTION IN WIRELESS SENSOR NETWORKS", International Journal of Computer Networks \& Communications (IJCNC) Vol.5, No.1, January 2013.

\section{Authors}

Lana Ibrahim Saeed, She is presently doing B.Sc. degree with honors in red sea university faculty of Engineering, complete her graduate research in September 2015. Her research interest on field of MANETs and Wireless Networks.

Hana Abd Almonem Osman, She is presently doing B.Sc. degree with honors in red sea university faculty of Engineering, complete her graduate research in September 2015. Her research interest on field computer and Wireless Networks.

Abdalla Osman, presently doing his B.Sc. degree with honors in red sea university faculty of Engineering, complete his graduate research in September 2015. His research interest on field of computer Networks.

Elmustafa Sayed Ali Ahmed, received his M.Sc. degree in electronic engineering, Telecommunication in 2012, and B.Sc. (Honor) degree in electrical engineering, Telecommunication in 2008. He was a wireless networks (Tetra system, Wi-Fi and WiMax) engineer in Sudan Sea Port Corporation for four years and a head department of electrical and electronics engineering, faculty of engineering in Red Sea University for 
one year. He published papers on wireless communications and networking in peerreviewed academic international journals and book chapters in big data clouds and computer systems. His areas of research interest include MANETs, wireless networks, VANETs, image processing, computer networks, and Cloud computing. 